\title{
Quantum register based on coupled electron spins in a room-temperature solid
}

\author{
P. Neumann ${ }^{1 \star}$, R. Kolesov ${ }^{1 \star}$, B. Naydenov ${ }^{1}$, J. Beck ${ }^{1}$, F. Rempp ${ }^{1}$, M. Steiner ${ }^{1}$, V. Jacques ${ }^{1}$, \\ G. Balasubramanian' ${ }^{1}$, M. L. Markham ${ }^{2}$, D. J. Twitchen ${ }^{2}$, S. Pezzagna ${ }^{3}$, J. Meijer ${ }^{3}$, J. Twamley ${ }^{4}$, \\ F. Jelezko ${ }^{1 \dagger}$ and J. Wrachtrup ${ }^{1 \dagger}$
}

Devices that harness the laws of quantum physics hold the promise for information processing that outperforms their classical counterparts, and for unconditionally secure communication'. However, in particular, implementations based on condensed-matter systems face the challenge of short coherence times. Carbon materials ${ }^{2,3}$, particularly diamond ${ }^{4-6}$, however, are suitable for hosting robust solid-state quantum registers, owing to their spin-free lattice and weak spin-orbit coupling. Here we show that quantum logic elements can be realized by exploring long-range magnetic dipolar coupling between individually addressable single electron spins associated with separate colour centres in diamond. The strong distance dependence of this coupling was used to characterize the separation of single qubits $(98 \pm 3 \AA$ ) with an accuracy close to the value of the crystal-lattice spacing. Our demonstration of coherent control over both electron spins, conditional dynamics, selective readout as well as switchable interaction should open the way towards a viable room-temperature solid-state quantum register. As both electron spins are optically addressable, this solid-state quantum device operating at ambient conditions provides a degree of control that is at present available only for a few systems at low temperature.

One of the greatest challenges in quantum information technology is to build a room-temperature scalable quantum processor ${ }^{7}$. Isolated electron and nuclear spins in solids are considered to be among the most promising candidates for qubits in that respect ${ }^{3,8}$. Several benchmark experiments including entanglement and elements of quantum memory ${ }^{9}$ have been achieved with spin ensembles, but ultimate functionality requires encoding quantum information into single spins. This however creates serious challenges in readout, addressing and nano-engineering single-spin arrays. The availability of photon-assisted single-spin readout ${ }^{10,11}$ and the possibility to create single defects by ion implantation ${ }^{12,13}$ make nitrogen-vacancy defects in diamond one of the most promising candidates in this respect. Paramagnetic nuclei in the vicinity of the electron spin can be used as auxiliary qubits with even more favourable relaxation properties ${ }^{14}$. As a consequence, coherence between electron and nuclear spin qubits has been exploited for showing all basic elements of a room-temperature quantum register ${ }^{5,15-17}$. The size of these registers however is limited to a few quantum bits owing to the limited number of nuclear spins that can be addressed in frequency space ${ }^{15,18}$. A critical step towards scalability is to develop a technique enabling mutual coupling of individual optically addressable quantum systems.
The system used in this study is a pair of single electron spins associated with separate nitrogen-vacancy defects in diamond. A single defect consists of a substitutional nitrogen atom in the diamond lattice and an adjacent vacancy (Fig. 1a,b). The electron spin triplet ground state of the defect shows a spin-dependent fluorescence emission enabling measurements of the spin state of single dopants using highly sensitive fluorescence microscopy techniques ${ }^{10,14}$. Recent experiments showing that nitrogen-vacancy defects can be coupled to spins of substitutional nitrogen have highlighted avenues for extending the scalability potential of the diamond quantum register ${ }^{6,19-21}$. In general, magnetic dipolar coupling can be used as a mechanism enabling the generation of entanglement in cases where coupling prevails over decoherence. The spin Hamiltonian describing the dipole-dipole interaction between two coupled spins is

$$
H_{\mathrm{dip}}=\frac{\mu_{0} g^{2} \mu_{\mathrm{B}}^{2}}{4 \pi r^{3}}\left[\hat{S}_{\mathrm{NV} \mathrm{A}} \cdot \hat{S}_{\mathrm{NV} \mathrm{B}}-3\left(\hat{S}_{\mathrm{NV} \mathrm{A}} \cdot \mathbf{r}\right)\left(\hat{S}_{\mathrm{NV} \mathrm{B}} \cdot \mathbf{r}\right)\right]
$$

where $\mu_{0}$ is the magnetic permeability, $g$ is the electronic $g$ factor, $\mu_{\mathrm{B}}$ is the Bohr magneton, $r$ is the distance between spins and $\mathbf{r}$ is the unit vector connecting them. The last term defines the angular dependence of coupling between nitrogen-vacancy defects, and the prefactor is about $70 \mathrm{kHz}$ for $r=10 \mathrm{~nm}$ (these values are relevant to the experiments presented in this letter). Such a distance between two colour centres enables their individual addressing using modern nonlinear microscopy techniques such as stimulated emission depletion microscopy ${ }^{22}$ (STED). At the same time, coupling within a few tens of kilohertz range might seem to be too weak to be detectable owing to decoherence, which is always present in solids. However, in diamond material with a reduced concentration of paramagnetic ${ }^{13} \mathrm{C}$ spins, coherence times can reach milliseconds at room temperature ${ }^{4}$. This letter presents the first step towards the realization of an optically addressable quantum register by demonstrating coherent coupling between two single nitrogen-vacancy defects engineered in such an ultrapure crystal.

Colour centre pairs were created by implanting patterns of high-energy nitrogen ions within small spots into synthetic isotopically enriched ${ }^{12} \mathrm{C}$ diamond. Figure $1 \mathrm{c}$ shows a pattern of implanted defects having two defects per spot on average. The implantation sites were investigated by measuring the fluorescence autocorrelation function $g^{2}(\tau)$ and ground-state depletion microscopy $y^{22}$, selecting a pair of colour centres with a distance of less than $30 \mathrm{~nm}$. As individual addressing by STED

\footnotetext{
${ }^{13}$ Physikalisches Institut, Universität Stuttgart, Stuttgart, 70550, Germany, ${ }^{2}$ Element Six Ltd, King's Ride Park, Ascot SL5 8BP, UK, ${ }^{3}$ Central Laboratory of Ion Beam and Radionuclides, Ruhr Universität Bochum, 44780, Germany, ${ }^{4}$ Centre for Quantum Computer Technology, Macquarie University, Sydney 2109 , Australia. ${ }^{*} T h e s e$ authors contributed equally to this work. ${ }^{\dagger}$ e-mail: f.jelezko@physik.uni-stuttgart.de; wrachtrup@physik.uni-stuttgart.de.
} 

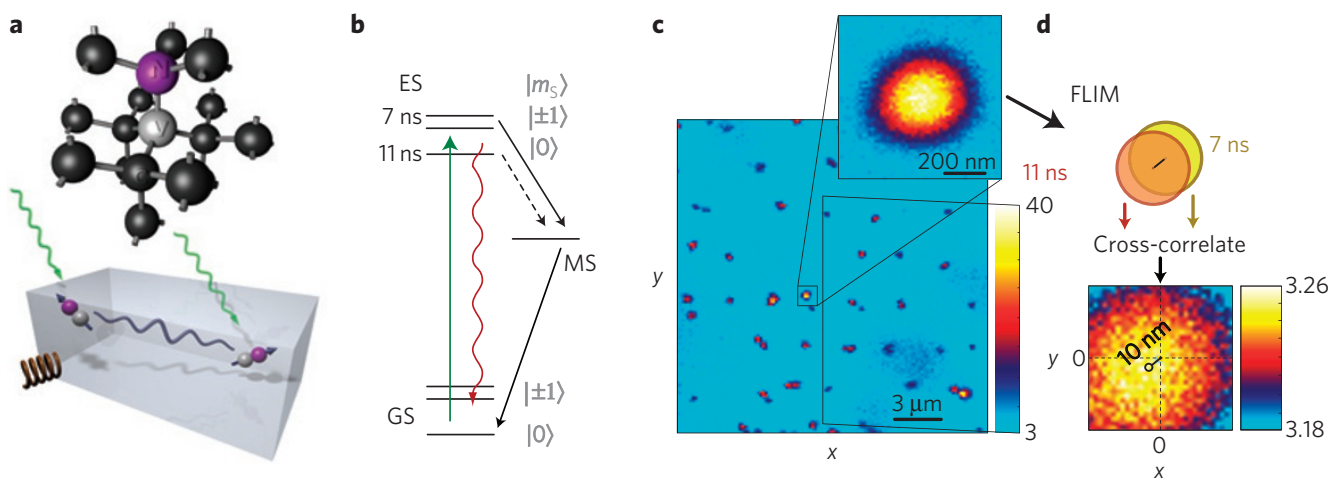

Figure 1 | Optical microscopy of coupled defects in diamond. a, Bottom: Illustration of two coupled nitrogen-vacancy defect centres in diamond manipulated by green laser light $(532 \mathrm{~nm})$ and microwave radiation. Top: Structure of the nitrogen-vacancy defect within the diamond lattice showing nitrogen (N) and carbon (C) atoms and the vacancy (V). b. Energy-level scheme of the nitrogen-vacancy colour centre comprising ground-state (GS) and excited-state (ES) triplets and a metastable singlet state (MS). Excitation light is shown as green arrows and fluorescence as a red arrow. Spin-dependent intersystem-crossing rates (black arrows) lead to spin polarization into the $m_{S}=0(|0\rangle)$ level, resulting in a higher fluorescence level for $|0\rangle$. c, Fluorescence microscopy image of the pattern of defects created by high-energy implantation of nitrogen. The detailed view shows the yet unresolved nitrogen-vacancy centre pair. The colour bar indicates the fluorescence intensity response in $10^{4}$ photons $\mathrm{s}^{-1}$. d, Using FLIM, two sub-images (schematic circles) are obtained from c, each belonging to a colour centre with a specific fluorescence lifetime (11 ns (orange), $7 \mathrm{~ns}$ (yellow)). The sub-images do show a significant overlap, but by cross-correlating the two images, a distance map is obtained (lower picture) showing a maximum for a separation of the two centres of $\approx 10 \mathrm{~nm}$. The colour bar indicates the cross-correlation result in $10^{4}$ (photons $\left./ 128 \mathrm{ps}\right)^{2}$. See Supplementary Information for more details.

exceeds our present experimental capabilities, fluorescence lifetime imaging (FLIM) analysis was used to resolve the two defects optically. In this approach, we use a contrast mechanism based on different decay rates of the excited-state spin sublevels ${ }^{23}$, enabling us to unravel the individual point-spread functions corresponding to the emission of the two colour centres. Note that FLIM is used here solely as a tool to determine the lateral distance of potential pairs of nitrogen-vacancy centres by purely optical means. It lacks the potential to interrogate one nitrogen-vacancy without touching the other as STED could do. Figure 1d shows the image of two resolved defects with the lateral distance of $8 \pm 3 \mathrm{~nm}$. At such short distances, the dipolar interaction between these two electron spins is expected to be observable in the magnetic resonance spectral response of the spins as shown below.

The electron spin transition frequencies of the colour centres are different when their symmetry axes have a different orientation with respect to the magnetic field as in the present case. This enables their individual addressing for a magnetic field of $\approx 43 \mathrm{G}$ applied parallel to one nitrogen-vacancy (Fig. 2a).The electron spin resonance spectrum can be described by the effective groundstate spin Hamiltonian

$$
H=\Sigma_{i=\mathrm{NV} \mathrm{A}}^{\mathrm{NV} \mathrm{B}}\left(\hat{S}_{i} \underline{\underline{\mathbf{D}_{i}}} \hat{S}_{i}+\mu_{\mathrm{B}} g_{e} \underline{\mathbf{B}} \hat{S}_{i}\right)+H_{\mathrm{dip}}
$$

Here $\mathbf{D}_{\mathrm{B}}$ are the zero-field splitting tensors, which mainly split $m_{\mathrm{S}}^{\mathrm{A}, \mathrm{B}} \stackrel{\equiv}{=} 0\left(|0\rangle_{\mathrm{A}, \mathrm{B}}\right)$ and $m_{\mathrm{S}}^{A, B}= \pm 1\left(| \pm 1\rangle_{\mathrm{A}, \mathrm{B}}\right)$ levels by roughly $D=2.87 \mathrm{GHz}$ and which are differently oriented for the two centres. The Zeeman energy of the electron spins is expressed by the second term in the sum with the magnetic field $\underline{\mathbf{B}}$. The last term of equation (2), explicitly given in equation (1), describes the magnetic coupling between spins.

The most appropriate way to detect the static magnetic dipolar interaction is through spin-selective Ramsey experiments. To this end, one of the two electron spins (spin A) is brought into a coherent superposition of its two eigenstates $\left(|0\rangle_{\mathrm{A}}+|1\rangle_{\mathrm{A}}\right) / \sqrt{2}$. This superposition acts as a sensitive magnetometer enabling us to resolve the magnetic field created by the second distant spin $(\operatorname{spin} \mathrm{B})$ with $\omega_{\mathrm{A}}^{\text {Ramsey }}=\omega_{\mathrm{A}}^{\text {Larmor }}+\omega_{\mathrm{A}}^{\mathrm{dip}} \cdot m_{\mathrm{S}}^{B}$. Here, $\omega_{\mathrm{A}}^{\text {Larmor }}$ is the Larmor frequency of spin $A$ and $\omega^{\text {dip }}$ is the dipolar coupling frequency of the spin pair. In the Fourier transformation analysis of the Ramsey

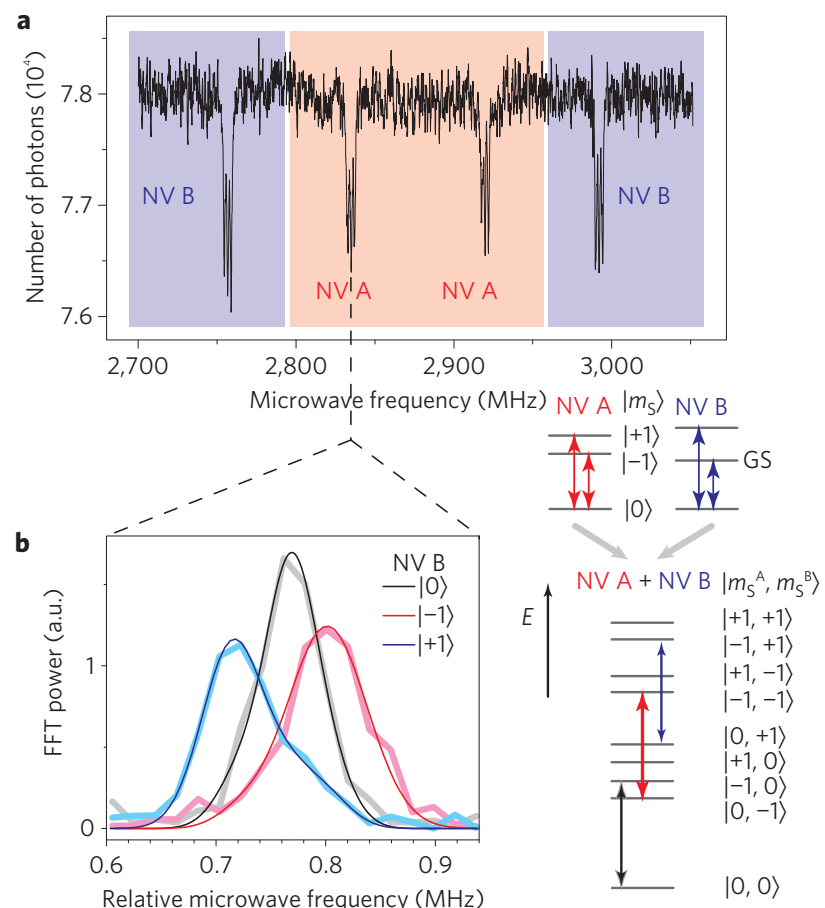

Figure 2 | Dipolar coupling between two single spins. a, Optically detected electron spin resonance spectra of two coupled defects showing fluorescence intensity $I_{\text {PL }}$ depending on the microwave frequency. $A$ magnetic field of $\approx 43 \mathrm{G}$ is applied parallel to nitrogen-vacancy $B(N V B$ ). Owing to different orientations in the lattice, single spins are addressable using spin-selective frequency pulses. The corresponding electron spin levels of the ground state of the nitrogen-vacancy centre are shown for both centres. $\mathbf{b}$, Shift of the spectral line of spin A depending on the state of spin $B$ mediated by magnetic coupling. The graph shows the fast Fourier transform (FFT) of the Ramsey fringes of spin $A$ after preparing spin $B$ in one of its eigenstates $(|-1\rangle,|0\rangle,|+1\rangle)$ by application of transition-selective microwave $\pi$ pulses. The spectral region shown is that of a single hyperfine line. Each line is fitted by three Gaussians corresponding to the three dominantly visible lines. The combined energy-level diagram shows the respective transitions. 
a
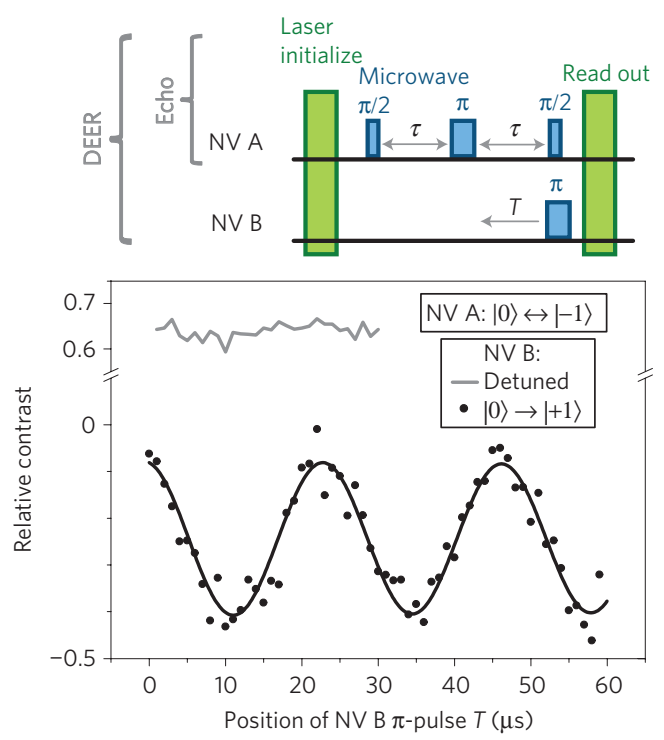

b

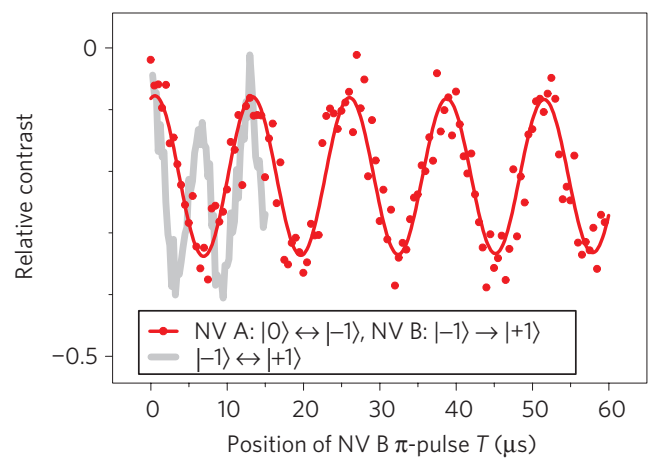

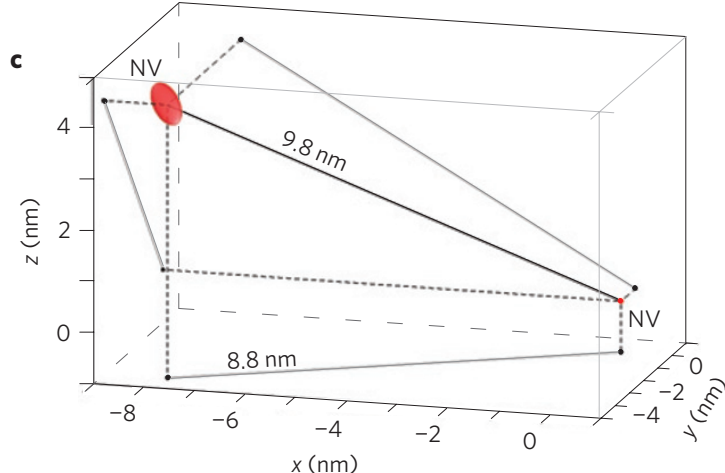

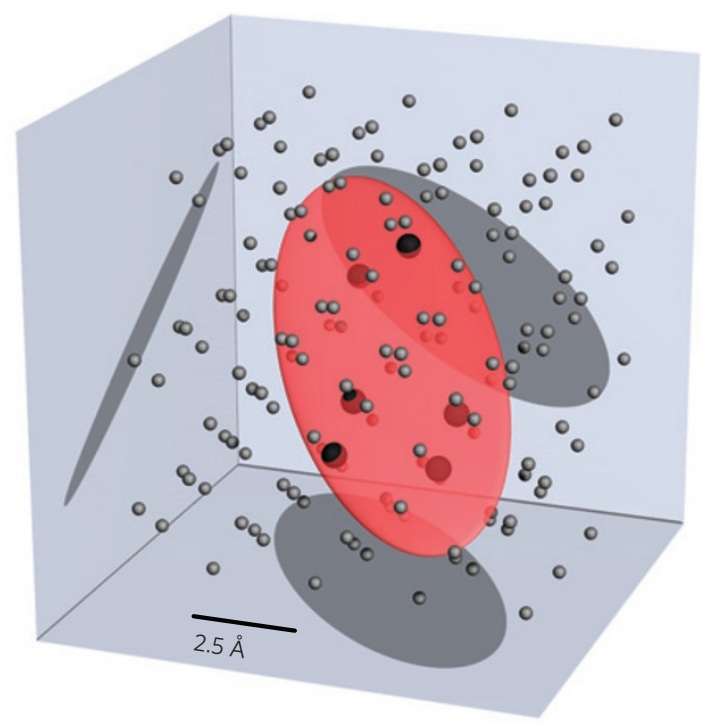

Figure 3 | Unravelling the structure of quantum register. a, Double electron-electron spin resonance (DEER) using microwave pulses selective between nitrogen-vacancy $A$ and $B$. Echo modulation is observed for spin $A$ when the coupled spin $B$ is flipped within the echo sequence. The modulation frequency corresponds to dipolar coupling strength $(42.7 \pm 0.4 \mathrm{kHz})$. A control experiment with a detuned driving field for spin B is also shown (grey line). Note that in the detuned case, nitrogen-vacancy $B$ is not flipped and thus the fluorescence intensity is higher. The vertical axis shows the relative fluorescence contrast normalized to the amplitude of a full Rabi oscillation of one nitrogen-vacancy centre. The zero value corresponds to one nitrogen-vacancy spin flipped to $| \pm 1\rangle$ but not the other one $(|0\rangle)$. This scaling is used for all pulsed measurements. b. Fast quantum gate operation using double quantum transition of spin B (red line, $(78.4 \pm 0.4 \mathrm{kHz}$ )) and both spins (grey line, $160 \pm 2 \mathrm{kHz}$ ). c, Reconstruction of two-qubit register geometry. Relative position of one spin with respect to the other positioned at the origin of coordinate system (upper part). The red area, which includes six possible lattice locations of the second spin, is defined on the basis of spin resonance measurements. A detailed view of the uncertainty area is shown in the lower part. Lattice sites are shown as grey spheres. The lattice locations inside the red area being identified as possible sites for the second nitrogen-vacancy are shown as bigger black spheres.

fringes (Fig. 2b), three different lines of spin A are visible that are related to three different eigenstates of spin $B|0, \pm 1\rangle_{\mathrm{B}}$, which we deliberately prepared by transition-selective microwave pulses. This is the first proof of magnetic dipolar coupling of the two spins. Besides showing coupling among qubits and their control, the initialization of the quantum register is of prime importance. As the relative intensity of coupling-induced lines is related to the accuracy by which either of the two spins is brought into a specific starting state, an estimation of the degree of efficiency of the initialization process can be made. By analysing the individual lines in Fig. $2 b$ in terms of contribution from the different $|0, \pm 1\rangle_{\mathrm{B}}$ states, we estimate that with a probability of $88 \pm 4 \%$, spin B is initialized into its $|0\rangle$ state (higher than the previously reported value for ensembles ${ }^{24}$ ). Note that our result is just a lower bound because it contains errors, for example, from imperfect $\pi$ pulses and misaligned magnetic fields, which lower the estimated value.

To determine the interaction of the two spins more accurately than the Ramsey fringe results from above and hence resolve their relative position in the lattice with great accuracy, it is best to use echo-based techniques that exploit the long phase-memory time $T_{2}$. Given the capability to separately address the two electron spins, a prominent way to measure the interaction between electron spins is based on a state-dependent phase-shift method similar to double electron-electron spin resonance used in electron spin resonance spectroscopy. In such a pulse sequence, a two-pulse echo measurement is carried out on spin $A$, whereas spin $B$ is flipped. The pulse sequence is designed so that all components of the $\underline{B}$ field acting on spin A, which are static during the pulse spacing time $\tau$, are refocused except the field originating from flipped spin B. The associated phase is $\Delta \varphi=\gamma \cdot \delta B \cdot T$, where $\delta B$ is the change in magnetic field at spin $A$ induced by spin $B$ and $\gamma$ is the gyromagnetic ratio. Hence, coupling causes a modulation of the signal as a function of time instant $T$ when the spin B is flipped (Fig. 3a).

This technique can be used for precise characterization of the relative position of the two spins. The result is shown in Fig. 3c, where we have placed the first spin at the origin of the coordinates system $(0|0| 0)$ (red dot), and the $x-y$ plane is parallel to the 
a
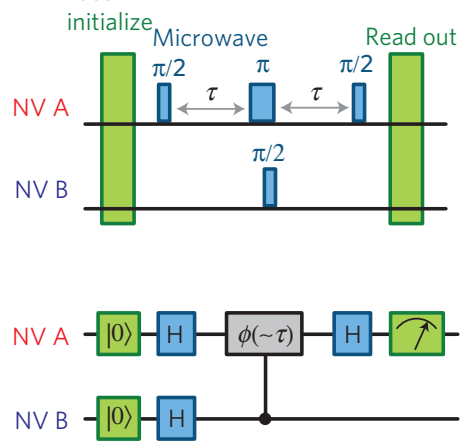

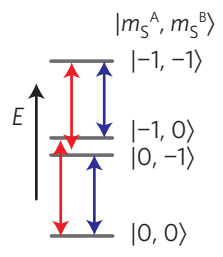

b

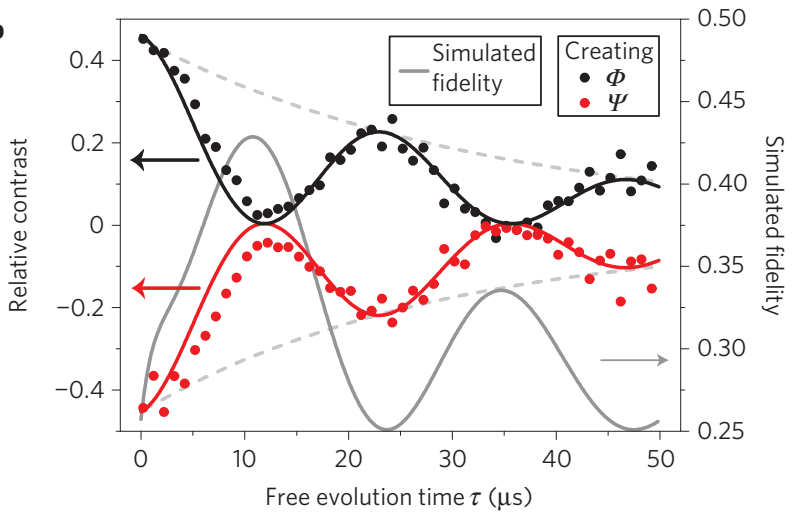

Figure 4 | Exploring dipolar coupling for creating an entangled state. a, Pulse sequence for generation of entanglement in the two-qubit quantum register $\left(|\phi\rangle\right.$-state in presented case). Preparing both centres in state $|00\rangle_{A, B}$, by optical pumping, an echo measurement is carried out on spin $A$. Right after the waiting time $\tau=\pi /(\gamma \cdot \delta B)$ and the echo's $\pi$ pulse on spin $A$, a $\pi / 2$ pulse on nitrogen-vacancy $B$ is applied resulting in state $(|-1,-1\rangle+i|-1,0\rangle+|0,-1\rangle$ $+|0,-1\rangle+i|0,0\rangle) / 2$. This is turned into $(-i|-1,-1\rangle+i|-1,0\rangle+|0,-1\rangle+|0,0\rangle) / 2$ after the second waiting time $\tau$. Finally, a $\pi / 2$ pulse on spin $A$ produces the quantum state $(i|-1,-1\rangle-|0,0\rangle) / \sqrt{2}$, which is the maximally entangled $|\Phi\rangle$ Bell state. b. Fluorescence intensity depending on pulse delay $\tau$ in entanglement sequence (red and black data points). The minima for the $|\phi\rangle$ (black) and the maxima for the $|\Psi\rangle$ states (red) mark the $\tau$ values of highest fidelity $\tau=(1,3,5, \ldots) \pi /(\gamma \cdot \delta B)$. The red and black curves are calculations using the coupling between the centres and their coherence times. The fidelity is simulated (grey curve) with identical parameters.

diamond surface. The location of the second spin is measured to be within the red ellipsoidal region (Fig. 3c, upper part), the size of which is defined by the experimental accuracy. Only six possible lattice positions fall within this area (Fig. 3c, lower part). The two qubits are measured to have a distance of $r=9.8 \pm 0.3 \mathrm{~nm}$ and a lateral separation with respect to the diamond surface of $8.8 \pm 0.3 \mathrm{~nm}$, which is in good agreement with the optical image analysis. Note that we have used the point-dipole approximation for calculation of spin-spin interaction. It has been shown that $\approx 90 \%$ of the electron spin density is located at the vacancy and its nearest-neighbour few-ångstrom-separated carbon atoms, which justifies the approximation.

Our experiments show that two qubits can be addressed and read out separately using transition-selective microwave fields. Moreover spin A and B can be coherently controlled. A final element necessary to complete the toolbox of quantum operations in our register is conditional spin dynamics among spin A and B. The spin flip executed on spin A in the experiment presented in Fig. $3 \mathrm{a}$ is transition dependent; therefore, the magnitude of the echo signal of spin A depends on the state of spin B. The fidelity of such a conditional spin flip is maximized after $\Delta \varphi=\pi$. Experimentally the observed fidelity is limited by the coupling strength of the two spins $(\approx 40 \mathrm{kHz})$ and the phasememory time of spin A $(110 \mu \mathrm{s})$. Moreover, because the nitrogenvacancy centre has spin $S=1$ (not $1 / 2$ ), double quantum transition $\left(\left|\Delta m_{l}\right|_{\mathrm{B}}=2\right)$ between the two states with extreme magnetic quantum numbers (here: $| \pm 1\rangle$ ) can be used for detecting the coupling. If spin $B$ is in state $|-1\rangle_{B}$ initially and then is excited to $|+1\rangle_{\mathrm{B}}$ the magnetic-field change $\delta B$ felt by nitrogen-vacancy $\mathrm{A}$ is doubled as compared with $\left|\Delta m_{I}\right|_{\mathrm{B}}=1$. According to $\Delta \varphi=\gamma \cdot \delta B \cdot T$, a doubled oscillation frequency is expected. Multiple quantum transitions were driven by composite microwave pulses. Furthermore, when in addition the echo measurement is carried out on the double quantum transition $|-1\rangle_{\mathrm{A}} \leftrightarrow|+1\rangle_{\mathrm{A}}\left(\left|\Delta m_{I}\right|_{\mathrm{A}}=2\right)$, a four times faster oscillation occurs (Fig. $3 \mathrm{~b}$ ) because the phase difference between these states is twice as sensitive for magnetic fields (and noise), as in the case of single quantum transitions.

Coherent coupling between spins is an important requirement for the creation of non-classical correlation in a two-qubit quantum register. The quantum circuit of this operation (Fig. 4a) uses controlled single qubits' operations realized by Hadamard gates and a two-qubit phase-shift gate. The observed signal oscillates between a maximum value corresponding to the quantum state $(i|0,-1\rangle-|0,0\rangle) / \sqrt{2}$ at $\tau=(0,2,4, \ldots) \pi /(\gamma \cdot \delta B)$ and a minimum value for the entangled state $|\Phi\rangle$ at $\tau=(1,3,5, \ldots) \pi /(\gamma \cdot \delta B)$ when the evolution period $\tau$ is varied (Fig. $4 \mathrm{~b})$. If the starting state is $|-1,0\rangle$, the oscillation is between states $(i|-1,-1\rangle-|-1,0\rangle) / \sqrt{2}$ and $(|-1,0\rangle+i|0,-1\rangle) / \sqrt{2}$, the latter one being the entangled state $|\Psi\rangle$. It is important to mention that the state preparation described above is computed for the ideal case (without taking into account decoherence). In our experiments, owing to the surprisingly short $T_{2}$ of nitrogen-vacancy B ( $\left.2 \mu \mathrm{s}\right)$, a random phase $\phi$ is acquired during state evolution. This fast decoherence of one of the spin qubits exceeds the coupling strength between the spins by an order of magnitude and limits the generation of entanglement. In fact, our results do show an entanglement fidelity, which is below 0.4. Different methods have been tested to lengthen $T_{2}$ of spin B such as decoupling of residual non-aggregated nitrogen atoms by dynamical decoupling sequences. However, none of them yielded the desired effect. From this we conclude that the short $T_{2}$ of spin B is not caused by a local 'spin bath' but rather is related to a yet unknown structural defect. Whereas values for $T_{2}$ in excess of $2 \mathrm{~ms}$ have been recorded for single nitrogenvacancy centres in isotopically enriched chemical-vapour-deposited diamond, the reduced values reported here for centres created using high-energy implants (see the Methods section) thus motivate further materials science research for detailed understanding and optimization of diamond-lattice dynamics in the presence of two closely spaced colour centres.

The results presented in this letter show a significant step towards the realization of a scalable room-temperature solid-state quantum information processing device. By artificially creating nitrogen-vacancy colour centres in ultrapure and isotopically engineered chemical-vapour-deposited grown diamond, we observe coupling of two single optically addressable qubits. Entanglement purification protocols ${ }^{25}$ using carbon nuclear spins as auxiliary 'storage' qubits can be explored for achieving high entanglement fidelities. Advanced single-atom fabrication technologies might further improve the engineering accuracy of the quantum register ${ }^{26}$. Most notably the coupling occurred over such long distances that state-of-the-art ultrahigh-resolution optical microscopy will enable the spins to be addressed separately. This will result in a highly versatile room-temperature quantum device. 


\section{Methods}

The diamond sample is an ultrapure type IIa synthetic crystal produced by microwave plasma-assisted chemical vapour deposition. The concentration of ${ }^{12} \mathrm{C}$ has been increased to $99.99 \%$ by using isotopically enriched methane in the growth process. Nitrogen-vacancy centres have been created by implantation of ${ }^{14} \mathrm{~N}$ ions with an energy of $18 \mathrm{MeV}$ and subsequent annealing at $800{ }^{\circ} \mathrm{C}$ for $2 \mathrm{~h}$. For this high implantation energy, the nitrogen to nitrogen-vacancy conversion efficiency is better than previously reported owing to a larger number of vacancies created (average nitrogen-vacancy creation efficiency 21\%). To increase the probability to create pairs of nitrogen-vacancy centres at small separations, several arrays of implantation sites with varying numbers of ions per spot have been created.

Nitrogen-vacancy centre pair candidates were detected by a home-built confocal microscope able to record optically detected magnetic resonance and the second-order correlation function. An adjustable electromagnet supplied a field of $30-80 \mathrm{G}$ in most experiments. Owing to the close proximity of the nitrogen-vacancy pair, the laser always excites both centres and their combined fluorescence response is collected in optically detected magnetic resonance experiments. High-resolution images were obtained by structured illumination microscopy and FLIM techniques to ensure that the distance of the nitrogen-vacancy centres allows for observation of magnetic dipole-dipole coupling. In the case of FLIM, a magnetic field of $\approx 700 \mathrm{G}$ was applied parallel to nitrogen-vacancy A's symmetry axis. All measurements have been carried out at room temperature.

Determining the relative position of two separate spins relies on the change of their coupling strength depending on the orientation of their quantization axes (equation (1)). These axes are changed by rotating the magnetic field. Eventually, recording the coupling frequencies for various magnetic-field settings by the echo-based techniques shown enables determination of the appropriate relative position of the two centres. See Supplementary Information for further information.

Received 16 July 2009; accepted 26 January 2010; published online 28 February 2010

\section{References}

1. Steane, A. Quantum computing. Rep. Prog. Phys. 61, 117-173 (1998).

2. Churchill, H. O. H. et al. Electron-nuclear interaction in C-13 nanotube double quantum dots. Nature Phys. 5, 321-326 (2009).

3. Trauzettel, B., Bulaev, D. V., Loss, D. \& Burkard, G. Spin qubits in graphene quantum dots. Nature Phys. 3, 192-196 (2007).

4. Balasubramanian, G. et al. Ultralong spin coherence time in isotopically engineered diamond. Nature Mater. 8, 383-387 (2009).

5. Childress, L. et al. Coherent dynamics of coupled electron and nuclear spin qubits in diamond. Science 314, 281-285 (2006).

6. Hanson, R., Dobrovitski, V. V., Feiguin, A. E., Gywat, O. \& Awschalom, D. D. Coherent dynamics of a single spin interacting with an adjustable spin bath. Science 320, 352-355 (2008).

7. Stoneham, M. Is a room-temperature, solid-state quantum computer mere fantasy? Physics 2, 34 (2009).

8. Hanson, R. \& Awschalom, D. D. Coherent manipulation of single spins in semiconductors. Nature 453, 1043-1049 (2008).

9. Morton, J. J. L. et al. Solid-state quantum memory using the P-31 nuclear spin. Nature 455, 1085-1088 (2008).

10. Gruber, A. et al. Scanning confocal optical microscopy and magnetic resonance on single defect centers. Science 276, 2012-2014 (1997).

11. Jelezko, F., Gaebel, T., Popa, I., Gruber, A. \& Wrachtrup, J. Observation of coherent oscillations in a single electron spin. Phys. Rev. Lett. 92, 076401 (2004).
12. Meijer, J. et al. Generation of single color centers by focused nitrogen implantation. Appl. Phys. Lett. 87, 261909 (2005).

13. Rabeau, J. R. et al. Implantation of labelled single nitrogen vacancy centers in diamond using N-15. Appl. Phys. Lett. 88, 023113 (2006).

14. Jelezko, F. et al. Observation of coherent oscillation of a single nuclear spin and realization of a two-qubit conditional quantum gate. Phys. Rev. Lett. 93, 130501 (2004).

15. Neumann, P. et al. Multipartite entanglement among single spins in diamond. Science 320, 1326-1329 (2008).

16. Dutt, M. V. G. et al. Quantum register based on individual electronic and nuclear spin qubits in diamond. Science 316, 1312-1316 (2007)

17. Mizuochi, N. et al. Coherence of single spins coupled to a nuclear spin bath of varying density. Phys. Rev. B 80, 041201 (2009).

18. Cappellaro, P., Jiang, L., Hodges, J. S. \& Lukin, M. D. Coherence and control of quantum registers based on electronic spin in a nuclear spin bath. Phys. Rev. Lett. 102, 210502 (2009).

19. Epstein, R. J., Mendoza, F. M., Kato, Y. K. \& Awschalom, D. D. Anisotropic interactions of a single spin and dark-spin spectroscopy in diamond. Nature Phys. 1, 94-98 (2005).

20. Gaebel, T. et al. Room-temperature coherent coupling of single spins in diamond. Nature Phys. 2, 408-413 (2006).

21. Hanson, R., Mendoza, F. M., Epstein, R. J. \& Awschalom, D. D. Polarization and readout of coupled single spins in diamond. Phys. Rev. Lett. 97, 087601 (2006)

22. Rittweger, E., Wildanger, D. \& Hell, S. W. Far-field fluorescence nanoscopy of diamond color centers by ground state depletion. Europhys. Lett. 86, 14001 (2009).

23. Batalov, A. et al. Temporal coherence of photons emitted by single nitrogen-vacancy defect centers in diamond using optical Rabi-oscillations. Phys. Rev. Lett. 100, 077401 (2008).

24. Harrison, J., Sellars, M. J. \& Manson, N. B. Measurement of the optically induced spin polarization of N-V centres in diamond. Diam. Relat. Mater. 15, 586-588 (2006).

25. Pan, J. W., Simon, C., Brukner, C. \& Zeilinger, A. Entanglement purification for quantum communication. Nature 410, 1067-1070 (2001).

26. Schnitzler, W. et al. Deterministic ultracold ion source targeting the Heisenberg limit. Phys. Rev. Lett. 102, 070501 (2009).

\section{Acknowledgements}

This work was supported by the EU (QAP, EQUIND, NEDQIT, SOLID), DFG (SFB/TR21, FOR730 and FOR1482), NIH, Landesstiftung BW, BMBF (EPHQUAM, KEPHOSI) and the VolkswagenStiftung.

\section{Author contributions}

P.N., R.K., M.S., J.B., V.J., B.N. and F.J. carried out the experiments, D.J.T. and M.L.M. designed and carried out synthesis of diamond material, J.T. and F.R. carried out theory analysis of dipolar coupling of nitrogen-vacancy defects, J.M., B.N. and S.P. carried out implantation of single nitrogen-vacancy defects and P.N., J.W. and F.J. wrote the paper. All authors discussed the results, analysed the data and commented on the manuscript.

\section{Additional information}

The authors declare no competing financial interests. Supplementary information accompanies this paper on www.nature.com/naturephysics. Reprints and permissions information is available online at http://npg.nature.com/reprintsandpermissions. Correspondence and requests for materials should be addressed to F.J. or J.W. 